\title{
Thin endometrial lining during frozen embryo cycles: a case-control study of risk factors and natural history
}

\author{
Emily A. Jacobs, ${ }^{1}$ Karen M. Summers,${ }^{1}$ Amy E. Sparks, ${ }^{1}$ Bradley J. Van Voorhis ${ }^{1}$
}

Keywords: Thin endometrial lining, IVF outcomes, endometrial thickness, live birth

\section{Objective}

To identify predictors of thin endometrial lining in the first frozen embryo transfer cycles and to characterize the natural history of this condition over subsequent cycles.

\section{Design}

Retrospective case-control study

\section{Setting}

Single academic institution

\section{Patient(s)}

Cases $(n=29)$ were patients who had a standard hormonal endometrial preparation cycle (oral estrogen 6 $\mathrm{mg} /$ day in divided doses for a minimum of 12 days) and had their first frozen blastocyst embryo transfer (FET) delayed or cancelled due to thin endometrium $(<6 \mathrm{~mm})$ between the years of 2012 and 2018. Controls $(n=58)$ were the next two consecutive patients undergoing a standard hormonal preparation who did not have a thin endometrium $(\geq 6 \mathrm{~mm})$ and proceeded with their first FET.

${ }^{1}$ Division of Reproductive Endocrinology and Infertility, Department of Obstetrics and Gynecology,, University of lowa Hospitals and Clinics, lowa City, IA, 52242

Please cite this abstract as: Jacobs EA, Summers KM, Sparks AE, Van Voorhis BJ. Thin endometrial lining during frozen embryo cycles: a case-control study of risk factors and natural history. Proc Obstet Gynecol. 2021;10(2):Article 7[ 2 p.]. Available from: http://ir.uiowa.edu/pog/ Free full text article.

Corresponding author: Bradley Van Voorhis, Department of Obstetrics and Gynecology, University of lowa, 200 Hawkins Drive, lowa City, IA 52242, brad-van-voorhis@uiowa.edu

Copyright: (c) 2021 Jacobs, et al. This is an open-access article distributed under the terms of the Creative Commons Attribution License, which permits unrestricted use, distribution, and reproduction in any medium, provided the original author and source are credited. 


\section{MATERIALS AND METHODS}

Medical charts were reviewed and demographic data and pertinent medical history was extracted. Multivariable logistic regression was performed to determine predictors of inadequate endometrium in FET cycles. Types of interventions utilized and subsequent endometrial thickness in additional cycles were recorded and the cumulative delivery rate from the initial attempt at FET onwards was calculated and compared with Fishers exact test and Kaplan-Meier survival analysis.

\section{RESULTS}

\section{$\underline{\text { Risk factors }}$}

Multiple logistic regression analysis of factors related to thin endometrium indicated thinner fresh cycle endometrium and lower body weight were associated with thin endometrial lining in FET cycles. Specifically, for each additional 5 kilograms of weight, the odds of having an endometrial lining under 6 $\mathrm{mm}$ decreased by 0.845 (95\% Cl: $0.717-$ 0.995), and for every $1 \mathrm{~mm}$ increase in endometrial thickness in the fresh cycle, women were 0.659 times less likely to have a thin endometrium in the subsequent FET (95\% Cl: 0.521-0.835).

\section{Natural History}

The mean endometrial thickness during first FET was $4.9 \pm 1.1 \mathrm{~mm}$ in cases versus $9.5 \pm 2.6 \mathrm{~mm}$ in controls ( $p$-value $<0.001$ ). During first FET, 10/29 cases had their estrogen dose extended. All 10 were able to achieve an adequate endometrial lining and subsequently went on to have a FET with a live birth rate of $40 \%$ (compared to a live birth rate of $50 \%$ in controls). A second FET occurred in 22 cases and 20 controls, a third occurred in 6 cases and 9 controls and a fourth occurred in 3 cases and 3 controls. Overall, mean endometrial thickness for cases did not differ clinically based on intervention utilized $(7.1 \pm 1.6$ $\mathrm{mm}$ with extended duration of oral estrogen, $7.01 \pm 1.2 \mathrm{~mm}$ with the addition of vaginal estrogen, $5.53 \pm 2.2 \mathrm{~mm}$ with intramuscular estrogen and $6.94 \pm 1.9$ $\mathrm{mm}$ with low dose gonadotropin stimulation). There was no statistically significant difference in cumulative live birth rate between cases and controls (55\% vs $72 \%$ respectively, p-value 0.148 ), however cases underwent more cycles to achieve live birth (mean number of cycles 3.0 vs 2.1 in control group, pvalue 0.013 ).

\section{CONCLUSIONS}

This study shows that prognosis after a diagnosis of thin endometrial lining is favorable. Lower weight and thinner fresh cycle lining are predictors of thin endometrial lining in FET cycles. Most importantly, women with a diagnosis of thin endometrial lining have similar live birth rates as those with adequate endometrial lining, although their time to achieve live birth is slightly longer.

Presented at "The Challenge of Maternal Mortality," University of lowa Obstetrics and Gynecology Postgraduate Virtual Conference, 20 November 2020 